\title{
Effects of electrical injury on musculoskeletal system: a case report
}

\begin{abstract}
Introduction: Electric injury of severe nature causing death is known as electrocution. Surviving patients may develop short term or long- term effects on various organs including gangrene, slow necrosis of tissues, neural and psychiatric problems.
\end{abstract}

Case report: A 27 years young male got electric injury accidently, leading to gangrene of left forearm \& hand and multiple site slow progressive death of skin requiring below elbow amputation of left upper limb, repeated debridement and management by skin grafting and trans-positional flaps. He did not develop any systemic, neural or psychiatric abnormality.

Clinical message: Electric injury patients should be observed for slow development of soft tissue and muscle necrosis even if they don't have any obvious problem on early examination.
Volume II Issue 4 - 2019

\author{
NS Dhaniwala,' Swapnil Date,' Mukund \\ Naresh Dhaniwala ${ }^{2}$ \\ 'Department of Orthopedics, Datta Meghe Institute of Medical \\ Sciences, Jawaharlal Nehru Medical College \& AVBRH, India \\ ${ }^{2}$ Fellow in Arthroplasty, Lokmanya Hospital, India
}

Correspondence: NS Dhaniwala, Professor of Orthopedics, JNMC \& AVBRH, DMIMS, Sawangi Meghe, Wardha 442I07, India, Email nsdhaniwala@gmail.com

Received: August 15, 2019 | Published: August 30, 2019

Keywords: suicide, electrical appliances, degree of injury, blackish in color, semiconscious

\section{Introduction}

Electrocution is an uncommon accidental event seen as an occupational hazard in electricity department field workers, more during rainy season and among electric pole workers. This can occur in general public in household settings also, when electric wiring is faulty or there is leakage of electricity due to short circuit and conduction of electricity through wet walls, metal taps, electric poles or electrical appliances. Rarely, electrocution may be chosen as a mode of committing suicide. In severe cases, death occurs instantly which is described as electrocution. But in many cases, the victim survives and develops short term or long-term ill effects on various systems of the body. Most of the electrical injuries are accidental and preventable.

Injury from manmade electrical power sources and lightning has been a hazard since its knowledge. The term electrical injury is used when it is not fatal but damage may cause dysfunction of multiple tissues or organs. ${ }^{1}$ Most industrial sources of electricity range from 0 $\mathrm{Hz}$ (Batteries) to $10 \mathrm{Kilo} \mathrm{Hz}$ (High tension power lines). Low voltage injuries $(<1000$ volts) are more fatal than high voltage injuries are. The blast effect of high voltage arcing can throw the victim away from the source, limiting the contact time. So, degree of injury can be small in high voltage injury. Low voltage injuries can cause muscle contraction leading to involuntary grip that prolongs the contact time with the current and increases the degree of injury. ${ }^{2}$ The case report herein describes effects of electric injury in relation to musculoskeletal system as seen in a 27 years old young man getting low voltage injury due to accidental contact.

\section{Case report}

A 27 years old male, JCB operator by occupation, accidently placed his left hand on the electric distribution panel of his residence early in the morning in half awake state. He got electrical injury and his left hand was in contact with the panel for an unknown period of time. Then probably he was thrown away or freed himself and fell down and became semiconscious. His relations saw him some time later, when he was trying to crawl and stand up. His left hand, wrist and forearm had become blackish in color and there was oozing of blood from his hand. The patient didn't remember the sequence of events of getting electrical injury and period soon after it. He was taken to a nearby hospital where his wounds were dressed and he was advised admission. The patient refused admission due to some important religious and family function. The patient soon noticed that his left upper limb below elbow is getting dry and darker in appearance and he is not able to move his wrist and hand to hold anything. He also felt that his left wrist and hand are bent towards palm in a very tight fashion. There were wounds on his both thighs, both feet, right hand \& forearm besides at the left elbow, which gradually started discharging some dirty brownish liquid. He got his wounds dressed at the local hospital and took advised medications but finding no relief, he came to our center for treatment 7 days after the electric injury.

Examination showed a thin built average nourished male patient without any systemic abnormality having charred appearance of left upper limb below elbow with full thickness burn of left hand and forearm extending $05 \mathrm{~cm}$ distal to left elbow crease. There was clear cut dry gangrene of left forearm and hand with established line of demarcation. There was death of superficial tissues in the cubital fossa which extended $03 \mathrm{~cm}$. proximally also. The wrist, metacarpophalangeal joints and interphalangeal joints of left-hand fingers were markedly fixed in flexion (Figure 1). There were full thickness scabs on both upper thighs, $(06 \mathrm{~cm}$. diameter on left thigh and $10 \mathrm{~cm}$ diameter over right thigh), right forearm distal $1 / 4^{\text {th }}$ and right hand first web space volar aspect (Figure 1B \& 1C) and blisters on dorsum of both feet dorsolateral aspect including $4 \& 5^{\text {th }}$ toes. The entry site of current was at left hand and the exit at both feet lateral 2 toes. The wounds' area was about $15 \%$ of the body surface area.

Patients haemoglobin was $15.3 \mathrm{gm} \%$, Alkaline phosphatase 154IU/L, SGOT 318IU/L, SGPT 685IU/L, total bilirubin 1.09 and $\mathrm{Hbs} \mathrm{Ag}$ was reactive. Other blood parameters were within normal limits. The patient's left forearm and hand was amputated through the line of demarcation without closing the wound. All the other wounds 
were debrided with complete eschar removal (Figure 2). The patient was put on injectable intravenous antibiotics with supportive drugs and regular wound care. The superficial wound on left cubital fossa, right distal forearm \& hand became deeper with further necrosis and needed repeated debridement. Wounds on both thighs were skin grafted after healthy granulation tissue formation. The wounds on both feet healed well, though left little toe amputation was needed due to dry gangrene. Wound on the right hand and forearm were managed with trans-positional myo-cutaneous flap from the local area by plastic surgeon. The patient continues to be under care of plastic surgeon. His repeat investigations, 1 month after admission, showed haemoglobin $13 \mathrm{gm} \%$, Alkaline phosphatase 65IU/L, SGPT 26IU/L, SGOT 20IU/L and total Bilirubin 0.2mg/DL, total Serum Protein 6.3gm/DL, Albumin 3.5gm/DL and Globulin 2.8gm/DL. Specimens from wounds showed growth of Oxacillin sensitive coagulase positive Staphylococci and Pseudomonas Aeruginosa sensitive to Amikacin, Ciprofloxacin and Vancomycin.

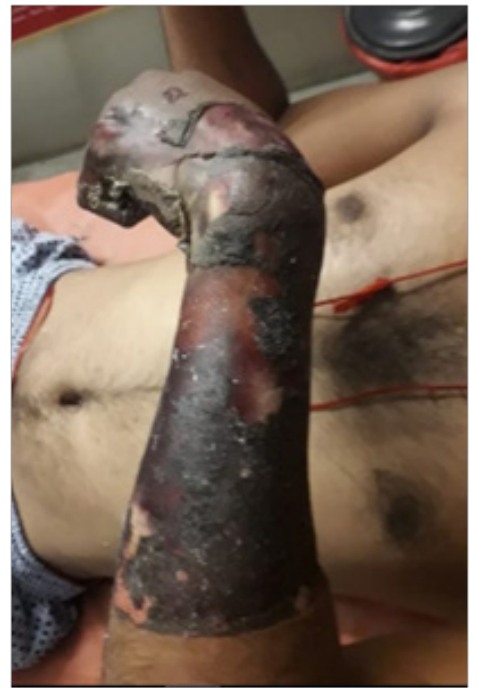

Figure IA Shows gangrenous left forearm and hand in flexed position.

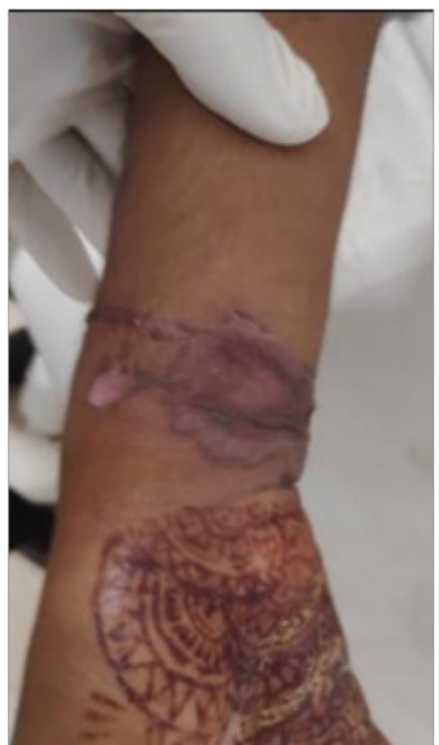

Figure IB Shows superficial wound on right distal I/4th forearm.

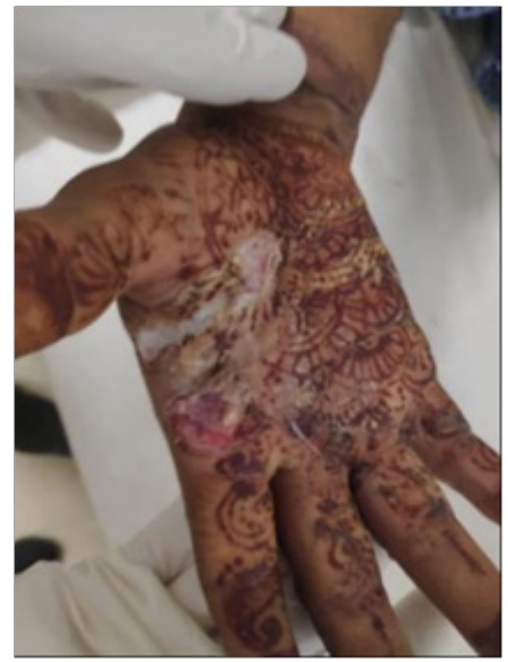

Figure IC Shows wound on the volar aspect of the right palm.
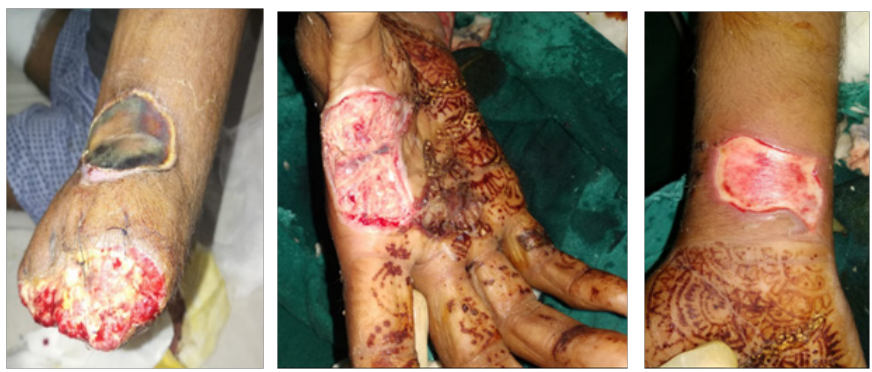

Figure 2 Shows amputated left forearm with gangrenous patch in cubital fossa, wound after debridement on right hand palm and right forearm.

\section{Discussion}

Four types of electrical injuries: flash, flame, lightning and true injury are described. Flash and flame cause superficial burns while lightning mostly leads to immediate death and charring of the whole body. True electrical injury makes an individual, part of an electrical circuit. In these cases, an entrance site and an exit site are usually seen. Half of all electrocutions in occupational setting occur due to contact with powerlines and about a quarter occur due to faulty electrical machines or tools. ${ }^{1}$

Electricity follows the path of least resistance through the body and creates heat resulting in thermal damage. Tissues with high resistance (bone, tendon, fat etc.) get more damage from heat. Skin, nerve and vessels have less resistance and may get less damage. Muscle has intermediate resistance but its greater volume in the body results in more passage of current and more injury. ${ }^{3}$ The human body has maximum (99\%) resistance on the skin. Wet skin, cuts and abrasions cause decrease in resistance. Skin acts as a capacitor and permits more current to flow if the voltage is changing rapidly.

Compared with general burn or thermal injuries, electrical injury can cause more damage to nerve, muscles, bone and skin with more complications and short- and long- term morbidity. Electricity has the ability to burn tissue beneath the skin even burning internal organs without apparent external evidence of it. ${ }^{4}$ Factors affecting the effect of electric injury are skin resistance, the voltage of current (Voltage $=$ current $\mathrm{x}$ resistance), the length of time of contact, the amount of electric current and its intensity. It disrupts normal operation of 
nervous and muscular systems and causes cell membrane damage across the length of tissue.

Electricity can cause involuntary contractions of muscles. Low frequency alternating current $(\mathrm{AC})$ tends to be much more dangerous than high frequency current. $\mathrm{AC}$ with the same ampere and voltage as Direct current (DC) is more dangerous and provokes worse effects. It causes tetany due to strong muscular contractions which induces "cannot let go" effect by freezing the muscles of the hand after the patient gets the shock. AC has a more tendency to induce heart fibrillation while DC causes cardiac arrest making the heart stop. Bradycardia, heart muscle injuries and arrhythmias may be produced because of electric injury. Walls of blood vessels through which a current has passed long enough may undergo necrosis.

Loss of consciousness, impaired recall, spinal cord injuries leading to weakness of lower limbs or all the four limbs, different cord syndromes and transient palsy have been reported as a result of electrocution. As long- term effect, post- traumatic stress disorder, depression, neuropathic pain may develop in patients. Variable degree of soft tissue damage progressing in a slow fashion, deep hole in the bone as part of exit wound, fracture or subluxations, compartment syndromes and muscle damage leading to necrosis can occur. ${ }^{4}$ Delayed neurological complications due to low voltage electrical injury are rarely reported. ${ }^{5,6}$ As long -term effect patient may develop depression and mild psychological effects requiring early detection and management. ${ }^{7}$

Very few reports of effects on musculoskeletal system are available in literature. Lee DH et al. ${ }^{8}$ reported on electrical injuries of the hand and upper extremity. Literature search shows articles on electrical injuries patho-physiology and general description of its effects on body with more stress on neural and psychiatric aspects. The reported case got accidental burn from low voltage current and suffered entry wound in left hand along with severe damage leading to gangrene proximally up to below elbow level. He was saved as he was thrown away and suffered thermal damage to both thighs, right forearm and hand and exit wounds at both feet. He still has loss of the memory regarding the event and mild depression. His blood parameters were within normal range except raised liver enzymes, which soon settled to normal levels. Gradual progression of tissue damage in limbs, requiring repeated debridement and skin coverage procedures indicate the early after effects of electric current. Haemoglobin, urine analysis, serum electrolyte, creatinine and creatinine kinase estimation are suggested for muscle damage evaluation and detection of anemia and kidney involvement. ${ }^{1}$ The patient does not have any neural deficit till date and continues under our follow up.

\section{Clinical message}

Electric injury can cause variable degree of gangrene, tissue necrosis, neurological damage and long-term neuropsychiatric problems in surviving patients. Besides, proper management of wounds, through and repeated evaluation of patient are needed for early diagnosis and management.

\section{Acknowledgments}

None.

\section{Conflicts of interest}

The authors declare there is no conflict of interest.

\section{References}

1. Zemaitis MR, Foris LA, Lopez RA, et al. Electrical injuries (Updated 2019 Jul 3). Treasure Islands, Florida: StatPearls Publishing; 2019.

2. Wesner ML, Hickie J. Long term sequelae of electrical injury. Can Fam Physician. 2013;59(9):935-939.

3. Tredgel EE, Shankowski HA, Tilley WA. Electrical injuries in Canadian burn care- identification of unsolved problems. Ann N Y Acad Sci. 1999;888:75-87.

4. Physiological effect of electric current. Wiki Lectures.

5. Andrews J, Reisner AD. Neurological and neuropsychological consequences of electrical and lightening shock: review and theories of causation. Neural Regen Res. 2017;12(5):677-686.

6. Kim HM, Ko YA, Kim JS, et al. Neurological complication after low voltage electric injury: A case report. Ann Rehabil Med. 2014;38(2):277281.

7. Andrews CJ, Reisner AD, Cooper MA. Post electrical or lightning injury syndrome: A proposal for an American Psychiatric Association's diagnostic and statistical manual formulation with implication for treatment. Neural Regen Res. 2017;12(9):1405-1412.

8. Lee DH, Desai MJ, Gauger EM. Electrical injuries of the hand and upper extremity. J Am Acad Orthop Surg. 2019;27(1):e1-e8. 